\title{
Balkan briefing. Reflections on the health of a refugee population (part 1)
}

\author{
Celia Helen Duff
}

During the early spring of 1999 the ethnic cleansing of Albanians from Kosovo saw the mass movement of a population from its country. Hundreds of thousands of people were evicted from their homes, pouring across the borders of neighbouring countries seeking sanctuary in hastily established refugee camps.

This article describes the health of this population and some of the immediate problems facing the humanitarian and health relief efforts encountered in the early stages of this massive crisis. The article is written from personal observation and is not based on formal research. It comments neither on policy nor on operational issues.

The author spent three weeks working with the United Kingdom team deployed to Macedonia to implement the plan for humanitarian evacuation. Staff from the Home Office made up this team with the addition of one medical officer representing the Department of Health. The role of the medical officer was, in part, to understand the health issues and to report back in preparation for receiving refugees into this country.

\section{Health before the exodus}

Until some 18 months before the recent problems in Kosovo, health service provision had been adequate for all with community provision of primary care services, dental care, immunisation programmes, etc. Specialist care, for example cardiothoracic surgery and haematology/oncology, was provided at a tertiary level by reciprocal agreement with other countries of the former Yugoslavia.

During the past 18 months these services had been progressively withdrawn from ethnic Albanians leaving a population unable to access some basic primary health care with a resultant decrease in their health status.

\section{The exodus}

The flight from their country was precipitate. Only the relatively fit, or those with relatives and friends able to shoulder an extra burden, were able to make the long journey to the borders. Many were left behind. A few could use motorised transport, most travelled on foot. Most were physically driven from their homes and subject to physical and psychological trauma during their eviction. With little or no time to prepare for their journey many of the chronically sick were unable to take with them the regular medication they might need. Clothing and equipment taken was also minimal: the weather at that time was cold and wet. Food and water was scarce; days were often spent waiting at a border crossing with access to neither.

\section{Immediate aftermath and associated health/welfare problems}

The health problems of this refugee population can be subdivided into three groups: those of any normal population, those relating to the flight from their homeland and those relating to the living conditions.

\section{NORMAL HEALTH PROBLEMS}

This population enjoyed good health and access to a full range of services before its expulsion. The incidence of cardiovascular disease was high, associated with a high prevalence of smoking. Drug abuse and promiscuity was low, this is a predominantly Muslim population. After the exodus, this population continued to experience its previous background morbidity with the complication of an almost complete lack of access to full health care and most particularly lack of access to secondary and tertiary care services.

HEALTH PROBLEMS RELATING TO THE EXODUS The health problems of these people resulting specifically from the forced departure from their country can be divided into two: physical and psychological.

The psychological problems cannot be underestimated. To the considerable stress of a long journey in appalling conditions, for many with the added responsibilities of young children and elderly relatives, must be added the trauma of the witness of torture and murder. The sheer necessity of survival during a journey will have pushed these realities mostly into the background but most who have suffered will need support for the inevitable resultant stress disorder before too long.

Physical problems will be associated with the nature of the journey, the lack of access to food and water, the lack of shelter and absence of basic sanitation. Significantly, many of the chronically sick, particularly importantly those with tuberculosis, insulin dependent diabetes, and those on chemotherapy, were unable to bring with them sufficient medication both for the journey and during the period of establishment of refugee support in camps.

Interestingly, there are apparently few immediately life threatening war related injuries requiring emergency medical evacuation. This may reflect the time between trauma and the international relief effort or the explanation may be simpler: that those injured did not make the journey across the border.

HEALTH PROBLEMS RELATING TO LIVING CONDITIONS

The refugee camps provide sanctuary and basic support for living and health needs. Each 
camp has a first aid/primary care post and some have a field hospital able to undertake major surgery. These services are provided by other nations and their length of stay is not yet agreed. Access to drugs and treatment is limited.

Tented accommodation provides overhead cover but will not shelter from extremes of wet or cold. The ground, formerly sodden from the April rains, has been covered with thick layers of sharp small rock and stone. Each tent has a supply of mattresses and blankets. There is barely space between each tent but wider walkways run between the rows.

Water is supplied through a series of standpipes that run from a central stored supply dependent on regular resupply from outside the camps. The water is fit for drinking. In most camps the daily food ration is given once a day from a central point. The diet never varies: white bread, tinned fish, soft cheese, oranges, fruit juice and milk for the children. Some smaller camps have field kitchens serving hot meals. The wealthy can supplement their diet from the myriad of small stalls set up within the camp perimeter.

Latrines are set on the edges of the camps: deep earth pits without hand washing facilities nearby. The larger camps have no other washing facilities: large plastic buckets make do as baths for the children and for a strip wash for the adults.

The footwear of most refugees is now in a pitiful state, many children have shoes that are now too small and some wear the shoes of their parents simply to protect their feet from the sharp ground. There is neither grass nor soft ground. Many of the refugees have only the clothes they arrived in: for some of the old this means wholly unsuitable wool or other thick garments.

The weather is getting extremely hot. There is little shade and drinking water is limited. The dust from the ground is pervasive and irritant to the eyes. The stones are bleached white from the sun and the glare can be unbearable at midday.

Community facilities for activity are limited. Some small camps have established schools and kindergartens. All have some play space for the teenagers: basketball courts, football pitches. Women busy themselves with the daily chores; the men sit in the shade and wait. Teenagers and children have found a peer group and developed their own society. All are bored and purposeless.

The old and disabled may be unable to move from their tents. Their relatives have no help in caring for them. They may be immobile and incontinent. Further groups posing additional problems are the mentally ill, for whom access to psychiatric services is non-existent, and the learning disabled.

\section{Ongoing health issues}

This population will need support for many months yet. While the basics are now in place there are a number of issues that will need tackling in the medium term. These include sanitation, nutrition, purposeful occupation, immunisation programmes for children, reestablishment of long term treatment for the chronically sick, supply of medication, access to secondary care and a method of identifying those needing specialist health care and providing for this. Longer term psychological support will also be necessary. Many of the children apparently belie the trauma of the past few months with a maturity beyond their years. This will not last.

Coordination of health care provision for these people is important, a task of some size given the number of agencies and nations involved. Local services are already becoming stretched and the supply of basic drugs, for example simple analgesia, is a problem. The number of refugees in Macedonia now makes up a significant proportion of the total population of that country. Apparent differences in access to care, for example free drug therapy and priority for secondary care for the refugees may cause local tensions.

\section{Conclusion}

This report has attempted to describe the health issues facing a population moved from its home. To these problems must be added the dimension of sustaining provision of health care over the long term while the crisis is resolved with the added complication of the impending winter. It is suggested that the inclusion of a medical officer with the Home Office team, one with a public health training, facilitated a more immediate understanding of the population health problems for those caught up in mass exodus from a home country. 